\title{
Three-Dimensional Matching based Resource Provisioning for the Design of Low-Latency Heterogeneous loT Networks
}

\author{
Ajay Pratap \\ ajaypratapf@mst.edu \\ Department of Computer Science \\ Missouri University of Science and Technology \\ Rolla, MO, USA. \\ Venkata Sriram Siddhardh Nadendla \\ nadendla@mst.edu \\ Department of Computer Science \\ Missouri University of Science and Technology \\ Rolla, MO, USA.
}

\author{
Federico Concone \\ federico.concone@unipa.it \\ Department of Computer Engineering \\ University of Palermo, \\ Viale delle Scienze, Palermo, Italy. \\ Sajal K. Das \\ sdas@mst.edu \\ Department of Computer Science \\ Missouri University of Science and Technology \\ Rolla, MO, USA.
}

\begin{abstract}
Internet-of-Things (IoT) is a networking architecture where promising, intelligent services are designed via leveraging information from multiple heterogeneous sources of data within the network. However, the availability of such information in a timely manner requires processing and communication of raw data collected from these sources. Therefore, the economic feasibility of IoT-enabled networks relies on the efficient allocation of both computational and communication resources within the network. Since fog computing and $5 \mathrm{G}$ cellular networks approach this problem independently, there is a need for joint resource-provisioning of both communication and computational resources in the networks. As the solution to this problem, we propose a novel three-dimensional matching based resource provisioning algorithm that minimizes average service latency in the presence of various resource constraints, task deadlines and non-identical preferences at IoT devices, fog access points (FAPs) and small-cell access points (SAPs) in 5G networks. We prove the stability and termination of the proposed algorithm and also demonstrate that our proposed algorithm outperforms other state-of-the-art algorithms through both, simulation and realworld experiments on the laboratory test-bed.
\end{abstract}

\section{CCS CONCEPTS}

- Mathematics of computing $\rightarrow$ Matchings and factors; $\bullet$ Networks $\rightarrow$ Network algorithms; Network resources allocation.

Permission to make digital or hard copies of all or part of this work for personal or classroom use is granted without fee provided that copies are not made or distributed for profit or commercial advantage and that copies bear this notice and the full citation on the first page. Copyrights for components of this work owned by others than ACM must be honored. Abstracting with credit is permitted. To copy otherwise, or republish, to post on servers or to redistribute to lists, requires prior specific permission and/or a fee. Request permissions from permissions@acm.org.

MSWiM '19, November 25-29, 2019, Miami Beach, FL, USA

(C) 2019 Association for Computing Machinery.

ACM ISBN 978-1-4503-6904-6/19/11...\$15.00

https://doi.org/10.1145/3345768.3355906

\section{KEYWORDS}

Resource Provisioning; PRB; 5G; IoT; Fog; SAP; Matching

\section{ACM Reference Format:}

Ajay Pratap, Federico Concone, Venkata Sriram Siddhardh Nadendla, and Sajal K. Das. 2019. Three-Dimensional Matching based Resource Provisioning for the Design of Low-Latency Heterogeneous IoT Networks. In 22nd Int'l ACM Conference on Modeling, Analysis and Simulation of Wireless and Mobile Systems (MSWiM '19), November 25-29, 2019, Miami Beach, FL, USA. ACM, New York, NY, USA, 8 pages. https://doi.org/10.1145/3345768.3355906

\section{INTRODUCTION}

Internet of Things (IoT) is a networking paradigm where ubiquitous smart-devices share information among each other to improve services in diverse applications such as smart grid, smart health, smart city, smart transportation and so on $[17,26]$. However, the ever-increasing deployment of low-cost IoT devices with limited computing capabilities introduces many computational challenges in the provisioning of services. In order to alleviate this problem, CISCO Systems, Inc. has developed a novel solution called fog computing, which offloads the computational load from the cloud to one/more fog access points (FAPs) that are closer to IoT devices $[3,23,24]$ within the network hierarchy. Consequently, there is an escalating growth in the demand for radio resources (e.g spectrum) to support communication between the ever-increasing number of IoT devices and FAPs. 5G cellular architecture is currently being developed to support the spectrum demand in IoT networks through dense deployment of small-cell access points (SAPs) (e.g. femtocell access point, picocell access points, microcell access points), along with Macro Base Station (MBS) within the cellular architecture $[4,12,14,19-22]$. This paper addresses the problem of resource provisioning in a novel IoT network architecture where fog computing and 5G cellular technologies are integrated together to simultaneously address limitations in both communication and computation.

The main challenge in designing intelligent services for IoT networks is to allocate resources across multiple heterogeneous 
entites in order to perform various kinds of tasks ${ }^{1}$, each having nonidentical, heterogeneous resource demands and stringent performance constraints (e.g. in terms of latency and/or quality of service) as shown in Table 1. For example, a driving aid service that leverages augmented reality technology relies on heterogeneous tasks such as object classification, tracking and recommender systems. However, such services demand significant computational capabilities which are not typically available at IoT devices. Although centralized architectures have necessary computational resources at the cloud/MBS, it is not always feasible to meet the stringent latency requirements, especially when the packets traverse through long queues and routes over the network. For example, even supremely advanced, geographically diverse gaming cloud server networks have difficulties guaranteeing application latency under $80 \mathrm{~ms}$ [6]. In order to overcome this limitation, we propose a fog-based architecture where tasks are off-loaded to nearby FAPs via availing necessary communication resources at SAPs, and delivered back accordingly to the respective IoT devices.

Motivating Appplication. Consider the problem of designing a smart health system where human activities are recognized based on information collected from various IoT devices (e.g. accelerometers and gyroscopes in mobile devices). For example, elderly people living in a retirement communities collapse frequently due to loss of balance. Therefore, a fall detection system helps mitigate latency by allowing appropriate healthcare personnel to attend their injuries on time. Similarly, sleep monitoring is another human activity recognition system needed to monitor and diagnose patients in a hospital. The raw data collected from the IoT devices and/or sensors have to be processed to extract relevant features for classification purposes. Since IoT devices have low computational capabilities and limited battery power, this data processing task needs to be performed by FAPs which provide real-time computational services on an on-demand basis. However, communication resources are needed to transfer information back and forth between IoT devices and FAPs, which are controlled by SAPs. In this paper, we design a novel architecture based on three-dimensional matching to accomodate the three types of agents, a.k.a. IoT devices, FAPs and SAPs, to address this resource allocation problem. For validation purposes, we consider a human activity recognition task in a smart health system to test the performance of our architecture.

Our Contributions. In this work, our goal is to minimize the average service latency at the IoT devices, via designing appropriate resource allocation strategies at FAPs and SAPs, in the presence of

${ }^{1}$ We refer to task as a basic unit of effort which cannot be further divided into sub-tasks.

\begin{tabular}{|l|l|}
\hline Applications & Latency \\
\hline \hline Home automation, video surveillance & 10 seconds \\
Web search, sensor readings & 1 second \\
Interactive web site, smart building, analytic & 100 milliseconds \\
Virtual reality, smart transportation & 10 milliseconds \\
Haptics, robotics, real-time manufacturing & 1 millisecond \\
\hline
\end{tabular}

Table 1: Applications and allowable latency. non-identical preferences at IoT devices, FAPs and SAPs. However, this introduces a contention with maximally allocating resources to increase the number of tasks served. Therefore, in order to serve this ill-posed problem, we propose a three-dimensional matching based algorithm to solve the above problem. We have also proved that our proposed algorithm converges to the stable matching solution in a fixed number of iterations. We have validated our proposed algorithm on data collected from real prototypes implemented in our laboratory.

The remaining of paper is organized as follows. Section 2 explains existing works. Section 3 includes system model. Section 4 describes three-dimensional matching algorithm for IoT-enable networks. Section 5 presents theoretical analysis. Section 6 presents a performance analysis based on both simulation and prototype based experiments. Section 7 concludes this work.

\section{RELATED WORK}

In [1], authors discussed a resource allocation problem in IoTenabled network. The proposed solution was applicable in assigning services to heterogeneous IoT demand model in the multiple network interfaces. The main drawback of this approach was slow convergence rate due to which this method is not suitable for densely deployed IoT networks [8]. In [29], a joint resource allocation and stable performance based model was studied. Authors, applied Stackelberg game and a many-to-many matching game model to maximize the utility of users, cloud and fog nodes. In [27], a joint radio and computational resource allocation problem is discussed in view of energy and latency requirements; and further a distributive method to solve the formulated optimization problem was proposed. The main drawback of this approach is to consider one centralized cloud service provider. The central point dependency may be obstacle in the case of expansion and elaboration of exiting infrastructure. In [16], authors proposed a three-dimensional matching based resource allocation technique for sensor and actuator model. The main drawback of this approach is stable matching convergence. The proposed scheme may not always guaranty a stable matching outcome in a large number of heterogeneous IoT devices.

In [15], a cost efficient resource allocation problem is formulated as an optimization problem and further double matching based algorithm proposed based on deferred acceptance algorithm from two-side matching to three-side matching. Convergence and computational complexity are major outfits of this approach. In [5], authors applied many-to-one version of deferred acceptance algorithm $[11,25]$ in-order to minimize the maximum gap between required and achieved latency for task execution in fog enabled networks. Authors did not talk anything about the radio resources availability and its allocation procedure which actually play a major part in task offloading to FAP. In [13], authors formulated joint radio and computational resource with respect to cost performance per user as an optimization problem. Further, a many-to-one matching based algorithmic model proposed to offload the task to FAP with help of cloud service provider. However, this approach is not suitable for critical deadline applications. Moreover, authors have assumed that all the users will share the radio and computational 


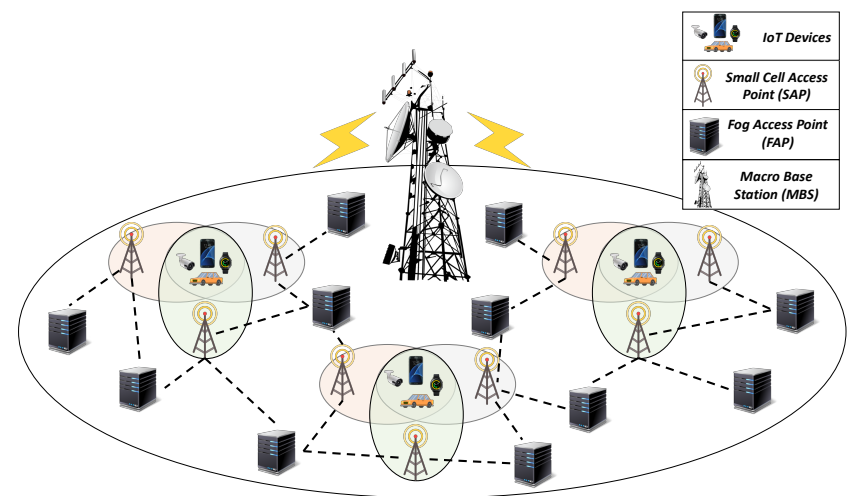

Figure 1: Heterogeneous IoT Networks

resources, equally regardless of data size and their deadline. So, equal distribution of resources do not justify the importance of different heterogeneous tasks and respective deadlines.

The existing approaches are not applicable in the three agents model where IoT, FAP and SAP will work altogether to achieve the heterogeneous task's deadline in limited available radio and computational resource of network. As each IoT device works independently regardless of others co-exiting tasks, it becomes very important to model these three agents altogether keeping deadline and limited availability of resources into consideration, and minimize the average service latency of task execution. Considering all these inhibitions of heterogeneous networks, in the following, we propose our system model followed by problem formulation.

\section{SYSTEM MODEL}

Consider a heterogeneous IoT network as shown in Fig. 1 which comprises of three types of agents: (i) IoT devices (which generate tasks), (ii) Small Cell Access Points, or SAPs (which control the communication resources), (iii) Fog Access Points, or FAPs (which control the computational resources), and (iv) a Macro Base Station or MBS (which moderates all the other agents in the network). Let $\mathbb{I}=\left\{I_{1}, \ldots, I_{K}\right\}, \mathbb{F}=\left\{F_{1}, \ldots, F_{R}\right\}$ and $\mathbb{L}=\left\{L_{1}, \ldots, L_{S}\right\}$ denote the sets of $K$ IoT devices, $R$ FAPs and $S$ SAPs respectively. In this paper, we assume that FAP, $F_{r}$, is equipped with $M_{r}$ processors running with a cycle rate of $c_{r}$ so that the total FAP's computational power is given by $\left(M_{r} \cdot c_{r}\right)$ processor cycles. Similarly, we assume that each SAP controls $N_{s}$ atomic resources for communication purposes called Physical Resource Blocks (PRBs) [10, 30], which comprises of $W=180 \mathrm{kHz}$ bandwidth and $0.5 \mathrm{~ms}$ time within a standard Orthogonal Frequency Division Multiple Access model [9].

When an IoT device generates a task, it will attempt to find the computational and communication resources needed to execute the task successfully. This search is driven by the IoT device's goal to minimize the service latency. The service latency $\Gamma_{k, r}^{s}$, due to the triplet $\left(I_{k}, F_{r}, L_{s}\right)$, mainly comprises of three components, as shown below:

$$
\Gamma_{k, r}^{s}=T_{\text {trans }}(k, r, s)+T_{\text {comp }}(k, r, s)+T_{\text {recv }}(k, r, s),
$$

where $T_{\text {trans }}(k, r, s)$ is the transmit time from IoT device $I_{k}$ to FAP $F_{k}$ on the PRBs assigned by $L_{s}, T_{c o m p}(k, r, s)$ represents the computation time of the task generated by $I_{k}$ at FAP $F_{r}$, and $T_{r e c v}(k, r, s)$ represents the receive time from FAP $F_{k}$ to IoT device $I_{k}$ on the PRBs assigned by $L_{s}$.

Let $x_{k, r}^{s}$ be a binary variable defined as follows:

$$
x_{k, r}^{s}= \begin{cases}1 & \text { if } L_{s} \text { allocates a PRB to }\left(I_{k}, F_{r}\right), \\ 0 & \text { otherwise. }\end{cases}
$$

Since the availability of PRBs is limited at any given SAP, we have the following allocation constraint at $L_{s}$ :

$$
\sum_{k=1}^{K} x_{k, r}^{s} \leq N_{s}
$$

for all $s=1, \cdots, S$.

Assuming that the data size is $D_{k}$, the transmit time $T_{\text {trans }}(k, r, s)$ in Equation (1) can be calculated as

$$
T_{\text {trans }}(k, r, s)=\frac{D_{k}}{\Upsilon_{k, r}^{s}},
$$

where

$$
\Upsilon_{k, r}^{s}=W \cdot N_{s} \cdot x_{k, r}^{s} \cdot \log _{2}\left(1+S I N R_{k, r}^{s}\right)
$$

is the maximum achievable data rate between IoT $I_{k}$ and FAP $F_{r}$. Here, $N_{s}$ is the total number of available PRBs at SAP $L_{s}$, and $S I N R_{k, r}^{s}$ is the signal-to-interference-plus-noise ratio ${ }^{2}$ between IoT $I_{k}$ and $F_{r}$ by using available PRBs at SAP $L_{s}$.

At FAP $F_{r}$, the compute time $T_{c o m p}(k, r, s)$ depends on the data size $D_{k}$, and the computational power of $F_{r}$, and can be calculated as follows:

$$
T_{\text {comp }}(k, r, s)=\frac{D_{k}}{y_{k, r}^{s} \cdot M_{r} \cdot c_{r}},
$$

where $y_{k, r}^{s}$ denotes the computational resource allocation to the pair $\left(I_{k}, L_{s}\right)$ as defined below:

$$
y_{k, r}^{s}= \begin{cases}1, & \text { if } F_{k} \text { allocates CPU to }\left(I_{k}, L_{s}\right) \\ 0, & \text { otherwise }\end{cases}
$$

Finally, we assume that the size of the task's outcome is significantly smaller ${ }^{3}$ than the original data size $D_{k}$. As a result, we neglect the contribution of receive time $T_{r e c v}(k, r, s)$ in the estimation of service latency $\Gamma_{k, r}^{s}$, and model $T_{r e c v}(k, r, s)$ as a random negligible time.

In this paper, our goal is to address the contention between minimizing the overall service latency

$$
U=\frac{1}{K} \cdot \sum_{s=1}^{S} \sum_{r=1}^{R} \sum_{k=1}^{K} x_{k, r}^{s} \cdot y_{k, r}^{s} \cdot \Gamma_{k, r}^{s},
$$

\footnotetext{
${ }^{2}$ For the sake of simplicity, we assume that the channel exhibits flat fading. However, this can be easily extended to frequency-selective fading channels as well.

${ }^{3}$ For example, in the case of binary classification of images, the outcome is a binary variable, whereas the input size $D_{k}$ is the size of the image itself.
} 


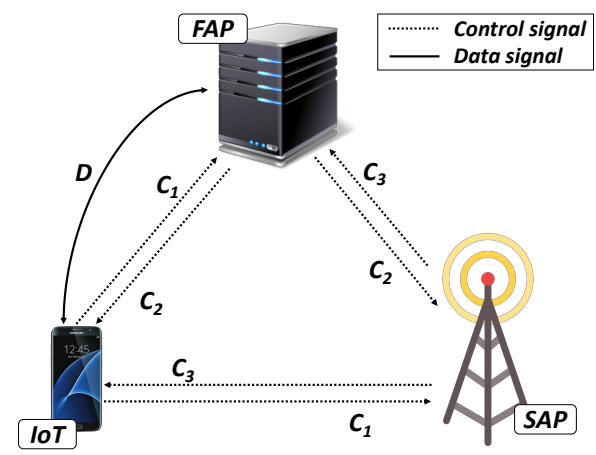

Figure 2: Network Interactions

and maximally allocating resources at both FAPs and SAPs, which is given by

$$
V=\frac{1}{K} \cdot \sum_{s=1}^{S} \sum_{r=1}^{R} \sum_{k=1}^{K} x_{k, r}^{s} \cdot y_{k, r}^{s}
$$

Due to the ill-posed nature of the problem, we model this interaction between different players (namely IoT devices, FAPs and SAPs) using matching, where appropriate triplets $\left(I_{k}, F_{r}, L_{s}\right) \in \mathbb{I} \times \mathbb{F} \times \mathbb{L}$ for all $I_{k} \in \mathbb{I}, F_{r} \in \mathbb{F}$ and $L_{s} \in \mathbb{L}$ are assigned to achieve the above stated goal. However, since each agent results in a specific preference ordering over two agents, this problem can not be solved using traditional two-sided matching approaches [2]. Since threedimensional matching is an NP-complete problem [18], we propose a novel three-dimensional pairwise matching in order to minimize the average service latency in the network.

\section{THREE-DIMENSIONAL MATCHING}

In this section, we propose the resource allocation algorithm based on three-dimensional matching of a triplet $\zeta=\left(I_{k}, F_{r}, L_{s}\right)$, where these three agents cooperate with each other to minimize service latency. We assume that the IoT devices, FAPs and SAPs are selfish in nature, and need to communicate with each other in order to reveal their preferences. In order to facilitate this interaction, we design the following communication protocol between the agents.

\subsection{Communications Protocol}

In this section, we present a communication protocol that supports necessary interaction between agents in forming a matching triplet $\left(I_{k}, F_{r}, L_{s}\right)$. As shown in Figure 2, whenever a task is generated at the IoT device $I_{k}$, it sends a control signal $C_{1}$ to FAP $F_{r}$ and SAP $L_{s}$, and requests necessary resources to complete the task. Upon receiving the control signal $C_{1}, F_{r}$ sends back another control signal $C_{2}$ to $I_{k}$ and $L_{s}$. At the same time, $L_{s}$ replies back to IoT and FAP using control signal $C_{3}$. Once this handshaking process is completed among the agents, the respective SAP allocates the required number of PRBs to the link IoT-FAP. Finally, respective IoT device receives the final result after the task at FAP is executed.

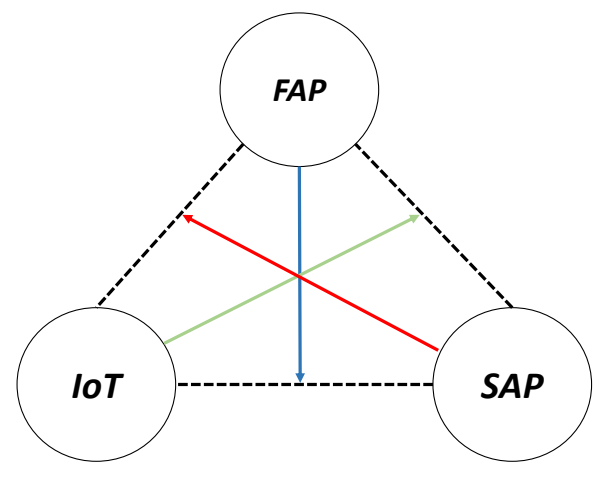

Figure 3: Preference model

\subsection{Construction of Preference Orders}

One of the main phases of the proposed algorithm is the construction of preference orders at each agent. Once a task is generated at the IoT device $I_{k}$, then it has a choice to team with any FAP-SAP pair. However, since IoT devices are concerned about minimizing service latency, it constructs a preference order over the set of all FAP-SAP pair based on the following relation:

$$
\left(F_{r}, L_{s}\right)>_{k}\left(F_{r^{\prime}}, L_{s^{\prime}}\right) \Leftrightarrow \Gamma_{k, r}^{s}<\Gamma_{k, r^{\prime}}^{s^{\prime}}
$$

Similarly, FAP constructs the preference order over the set of all IoT-SAP pairs based on the computational time required to complete the task. In other words, FAP follows these two rules while selecting the appropriate pairs. More specifically, the FAP $F_{k}$ can minimize the computational time of successful task allocations by (i) picking tasks in the order of decreasing computational load, and (ii) identifying those tasks that have a greater chance of PRB allocations. Therefore, FAP prepares a preference list based on the following relation:

$$
\left(I_{k}, L_{s}\right)>_{r}\left(I_{k^{\prime}}, L_{s^{\prime}}\right) \Leftrightarrow T_{\text {comp }}(k, r, s)<T_{\text {comp }}\left(k^{\prime}, r, s^{\prime}\right),
$$

which means FAP prefers the IoT-SAP pair to which computational latency for successful completion of task is less.

Similarly, SAP prepares the preference ordering of IoT and FAP based on transmission latency. In other words, SAP selects the IoTFAP link, which has higher achievable data rate and consequently it will result in lower transmission latency. The preference ordering of IoT-FAP link is prepared as per following expression:

$$
\left(I_{k}, F_{r}\right)>_{s}\left(I_{k^{\prime}}, F_{r^{\prime}}\right) \Leftrightarrow T_{\text {trans }}(k, r, s)<T_{\text {trans }}\left(k^{\prime}, r^{\prime}, s\right) .
$$

\subsection{Desired Properties of a Match}

Let $\mu$ denotes the match, which comprises of the set of all approved triplets at any given iteration. Furthermore, let $\zeta_{B}=\left(I_{k}, F_{r}, L_{s}\right) \in \mu$ be one of any approved triplets at the start of some intermediate iteration within the algorithm. If the IoT device $I_{j}(j \neq k)$ reveals its proposal to $\left(F_{r}, L_{s}\right)$ to include the triplet $\bar{\zeta}_{B}=\left(I_{j}, F_{r}, L_{s}\right)$ in $\mu$, contention arises between the current match $\mu$ and a potential match $\mu-\zeta_{B} \cup \bar{\zeta}_{B}$. This contention can be resolved based on the following blocking triplet concept: 
Definition 1. For any $j=1, \cdots, K$ and $k=1, \cdots, K$, a triplet $\bar{\zeta}_{B}=\left(I_{j}, F_{r}, L_{s}\right)$ blocks the triplet $\zeta_{B}=\left(I_{k}, F_{r}, L_{s}\right)$ from being included in $\mu$ if

(i) $U\left(\mu-\zeta_{B} \cup \bar{\zeta}_{B}\right)<U(\mu)$, and

(ii) $\left(I_{j}, F_{r}\right)>_{s}\left(I_{k}, F_{r}\right)$ and $\left(I_{j}, L_{s}\right)>_{r}\left(I_{k}, L_{s}\right)$.

This definition implies that if the IoT device $I_{j}$ results in lower average service latency than $I_{k}$ (Equation 8), by forming a triple with $\left(F_{r}, L_{s}\right)$, then $\left(I_{j}, F_{r}, L_{s}\right)$ forms a blocking triple and will replace $\left(I_{k}, F_{r}, L_{s}\right)$ in $\mu$ [18].

In this paper, we define stability of a three-dimensional match based on the blocking triplet concept.

Definition 2. A matching $\mu$ is stable if there exist no blocking triplet for $\mu$.

In the following section, our objective is to find the stable matching among IoTs, SAPs and FAPs in the networks.

\subsection{Proposed Algorithm}

The proposed three-dimensional matching algorithm found in Algorithm 1 takes the sets of IoT devices, FAPs with respective computational power, SAPs with respective available PRBs as input, and delivers a three-dimensional stable matching $\mu$ as an output. Broadly, the algorithm can be divided into two phases: (i) Preference List Estimation, and (ii) Matching.

In the Preference List Estimation phase, service latency $\Gamma_{k, r}^{s}$ is estimated for every possible triplet $\left(I_{k}, F_{r}, L_{s}\right) \in \mathbb{I} \times \mathbb{F} \times \mathbb{L}$, as shown in Line 1 . Note that the estimation of $\Gamma_{k, r}^{s}$ depends on the computation of both $T_{\text {comp }}$ and $T_{\text {trans }}$, which are needed to evaluate preference orders at both FAPs and SAPs respectively. Then, each agent prepares a preference list over the pair of other two agents, as shown in Equations (10-12), and reveals them to MBS, as stated in lines 3-5.

In the Matching phase, each unmatched IoT device $I_{k}$ sends a proposal to FAP-SAP pair $\left(F_{r}, L_{s}\right)$ on the top of its respective preference list in Line 8. As stated in Line 9, if MBS finds the pairs $\left(I_{k}, L_{s}\right)$ and $\left(I_{k}, F_{r}\right)$ in $\mathbb{F}_{\text {Pref List }}$ and $\mathbb{L}_{\text {Pref List }}$ (which are updated to include those pairs that were never evaluated in the past) respectively, then the triplet $\left(I_{k}, F_{r}, L_{s}\right)$ is included in the match $\mu$. However, if FAP $F_{r}$ or SAP $L_{s}$ or both are not listed in $\mathbb{F}_{\text {Pref List }}$ and $\mathbb{L}_{\text {Pref List }}$, two possibilities arise. In the first possibility, $\left(I_{k}, F_{r}, L_{s}\right)$ forms a blocking triplet, in which case, the corresponding triplet present in $\mu$ needs to be rejected as stated in Line 14 , and then replaced by $\left(I_{k}, F_{r}, L_{s}\right)$ in $\mu$. Alternatively, if $\left(I_{k}, F_{r}, L_{s}\right)$ does not form a blocking triplet to any other triplet in $\mu$, it is just rejected by MBS. The proposed algorithm terminates when all the IoT devices made requests to some FAP-SAP pair, i.e., either the IoT device is matched with appropriate FAP-SAP pair to be included in $\mu$, or it has been rejected by all FAP-SAP pairs.

\section{ANALYSIS OF PROPOSED ALGORITHM}

As the traditional three-sided matching is NP-complete problem, there is possibility that it would not converge to stable matching $\overline{\text { Algorithm } 1 \text { Three-Sided Matching based Resource Allocation }}$ Algorithm

Input: Set of IoT devices $\mathbb{I}$,set of FAPs $\mathbb{F}$ with respective computational power i.e., $M$ and $c$ and set of SAPs $\mathbb{L}$ with respective available PRBs i.e., $N$.

Output: A stable three-dimension matching $\mu$.

1: Phase 1: Preference List Estimation

2: Estimate $\Gamma_{k, r}^{s}$ based on Equation (1), for $I_{k} \in \mathbb{I}, F_{r} \in \mathbb{F}$ and $L_{s} \in \mathbb{L}$.

3: Each IoT device $I_{k}$, prepares the preference list over FAP-SAP agents based on Equation (10), and inform to MBS.

4: Each FAP, SAP prepare the preference list of IoT-SAP, FAP-IoT based on Equations (11) and (12), respectively and inform to MBS.

5: MBS prepares the preference lists $\mathbb{I}_{\text {Pref List }}=\left\{I_{k}\right\}_{k=1}^{K}$ for IoT devices, $\mathbb{F}_{\text {Pref List }}=\left\{F_{r}\right\}_{r=1}^{R}$ for FAPs, and $\mathbb{L}_{\text {Pref List }}=$ $\left\{L_{s}\right\}_{s=1}^{S}$ for SAPs based on above lines 5-6.

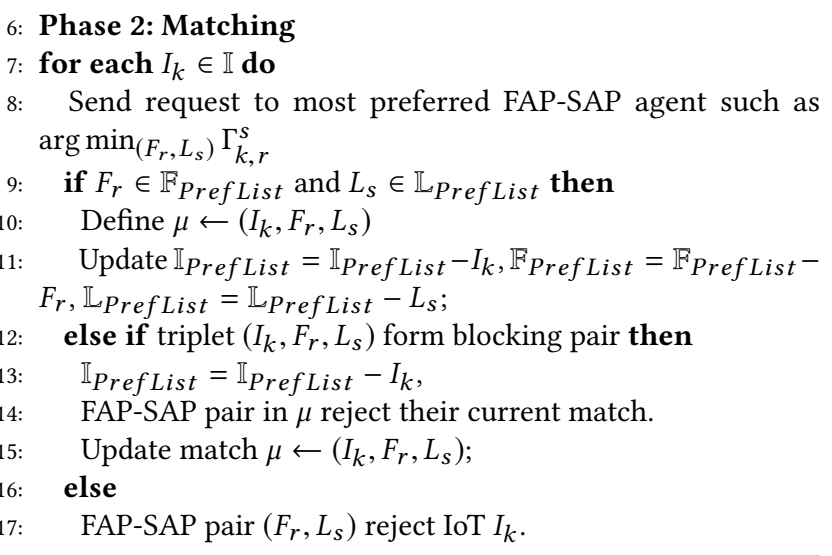

[28]. However, our proposed algorithm differs from the traditional three-sided matching problem in the sense that in our case agents are mutual, i.e., if computational time at FAP is less and data rate assigned by SAP between IoT-FAP is high, then the overall service latency of any IoT device will be low. In the other word, if computational and transmission latency of tasks are less then corresponding overall service latency will also be less. Thus, the preference lists of different agents in our proposed algorithm are mutual.

THeOREM 1. The proposed algorithm terminates after a finite number of iterations.

Proof. The proposed Algorithm 1 will terminate after a fixed number of iterations. We can prove as follows: (a) an IoT device sends a request to a FAP-SAP pair only once, (b) the total number of FAP-SAP pairs are fixed, i.e., $R \times S$. In each iteration an IoT device sends a request to FAP-SAP pair which has not rejected to this IoT device earlier. Thus, as the number of iterations increases, the remaining choices over FAP-SAP get reduced. An IoT device has $R \times S$ number of choices and the maximum number of trial can not go more than $R \times S$ in the worst case. In the other words, either IoT device matched to FAP-SAP pair and no other pair will make 


\begin{tabular}{|c|c|c|}
\hline Description & Symbol & Value \\
\hline \hline Task data size $(M B)$ & $D$ & $\{100,200, \ldots, 1000\}$ \\
Number of IoTs & $K$ & $\{10,20,30, \ldots, 300\}$ \\
Number of CPUs at FAP & $M$ & $\{2,4,8\}$ \\
CPU frequency $(G H z)$ of FAP & $c$ & $\{1.5,3,6,12,14\}$ \\
Number of PRBs & $N_{s}$ & $\{2,4,6,8,10\}$ \\
\hline
\end{tabular}

Table 2: Simulation Environment.

benefit in the service latency or IoT device not matched and all the FAP-SAP pairs have rejected it. Thus, the proposed algorithm will terminate after a finite number of iterations.

THeorem 2. The proposed algorithm converges to a stable match.

Proof. Based on above Theorem 1 we conclude that the proposed algorithm terminates after a fixed number of iterations. However, to prove that the outcome of the proposed algorithm is stable, we need to show that the final matching $\mu$ will not have any blocking pair. Based on Phase 1 of the proposed algorithm we can guarantee that there will not be any blocking pair in the matching $\mu$. Let's say a blocking triplet $\zeta_{B}=\left(I_{k}, F_{r}, L_{s}\right)$ exist in the final matching $\mu$. According to line 12 of our algorithm, we say whenever there is a blocking pair in the temporary matching, either the FAP-SAP pair will reject all their previous matched triplets or they reject the current IoT device request because of which blocking arises (lines 12-18). Thus, the outcome of the proposed algorithm will never have a blocking pair and this contradicts. Hence, the proposed algorithm converges to stable matching.

THEOREM 3. The time complexity of proposed algorithm is $O(K R S$ $\left.\log _{2}(R S)\right)$

Proof. Each IoT device prepares a preference ordering of FAPSAP pairs in Phase 1. Using heap sort algorithm an IoT device can sort the preference list in $\mathrm{O}\left(\mathrm{RS} \log _{2}(R S)\right)$ time complexity. As we have $K$ IoT devices thus, total complexity results as $O\left(K R S \log _{2}(\mathrm{RS})\right)$. However, in Phase 2, each IoT device proposes at most $R \times S$ agents and there are $K$ IoT devices, consequently time complexity results at $O(K R S)$. Thus, the total time complexity of proposed algorithm is concluded at $O\left(K R S \log _{2}(R S)\right)+O(K R S)$, i.e., $O\left(K R S \log _{2}(R S)\right)$.

\section{PERFORMANCE STUDY}

We validate our proposed algorithm using both virtual and realworld experiments. In the first case, we test our algorithm on simulated environments based on JAVA, which are then plotted using MATLAB. Later, we test our algorithm on a laboratory prototype using a smart watch as an IoT device, the hotspot in a smart phone as SAP and a laptop as a FAP. Details of all the experiments are given below.

\subsection{Simulation Analysis}

In order to evaluate the performance of the proposed algorithm, we have simulated a network model that consists of a random number of FAPs and SAPs ranging between 1 to 300, which are distributed uniformly over a geographical area. Each FAP is randomly assigned the number of processors, CPU frequency, and a non-zero number

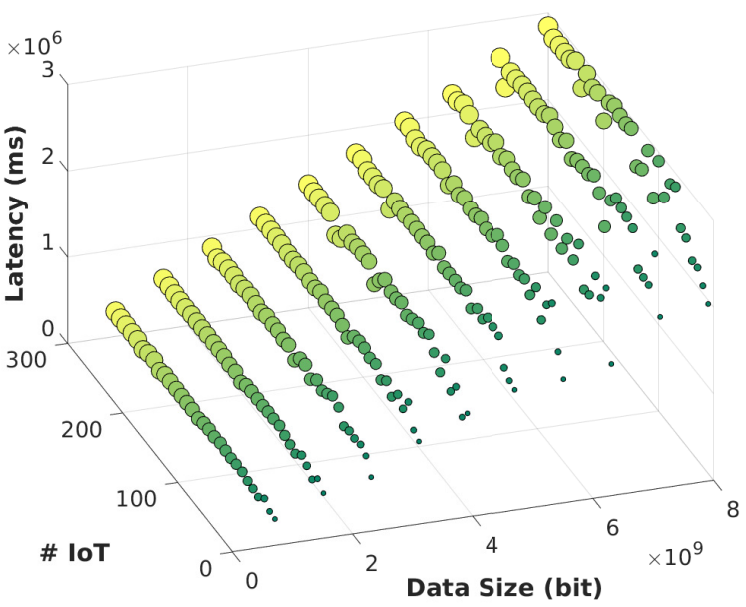

Figure 4: Latency of the system by varying the number of IoTs and the data size.

of SAP links. The number of available PRBs at each SAP is assigned randomly between 1 to 10 . In addition, we assumed that the number of IoT devices can vary from 10 to 300 and that each of them can generate tasks with data sizes ranging from $100 \mathrm{MB}$ to $1000 \mathrm{MB}$. Parameters used in the simulation are summarized in Table 2.

Service latency analysis: Fig. 4 represents the pattern of the average service latency while varying the number of IoT devices and data size. From the results we can conclude that with increase of data size service latency increases and with increase of number of IoT devices, there is a very slight variation in average latency. The reason is like, with increase of data size, transmission and computational latency increase, and consequently total service latency increases in the system.

Satisfaction analysis: In Fig. 5, we have shown a comparison between number of IoT devices, satisfaction and data size. Satisfaction of an IoT device under matching $\mu$ is defined as the rank (position) of the matched agents $\mu\left(I_{k}\right)$ in $I_{k}$ 's preference list as defined in the following:

$$
\text { Satisfaction }(\%)=100 \times \frac{\text { size }- \text { rank }}{\text { size }}
$$

where, size is the maximum size of preference list of any IoT device and rank is the position of matched agents. From the result, we can see that when number of IoT devices is less satisfaction is high, whereas with increase of number of devices on different data size, satisfaction becomes almost constant.

Execution time analysis: We examined the behavior of the algorithm execution time when the number of IoTs increases and the size of the data differs. From the result (Fig. 6), we can conclude that the total execution time grows with the number of IoT devices. This is because larger number of IoT devices result in longer preference lists, which in turn increases the time to compute a stable match among the three agents. However, the data size does not seem to have any effect on the execution time of different task sizes. 


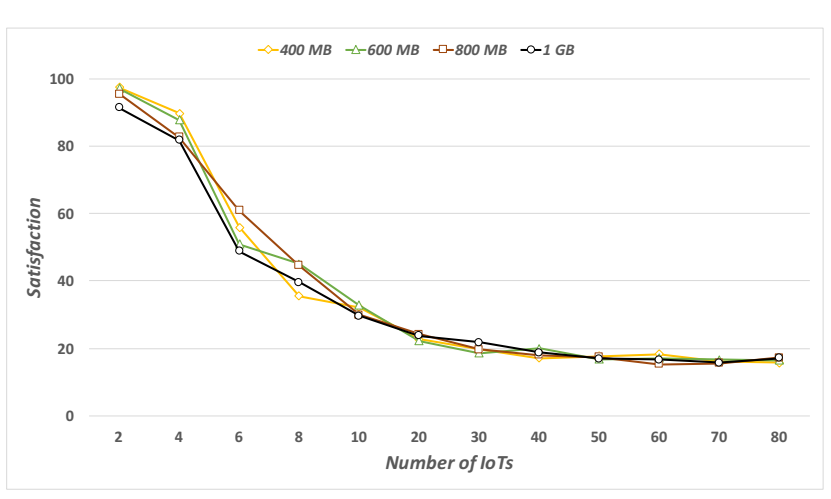

Figure 5: Comparison between number of IoT devices and satisfaction over different data sizes.

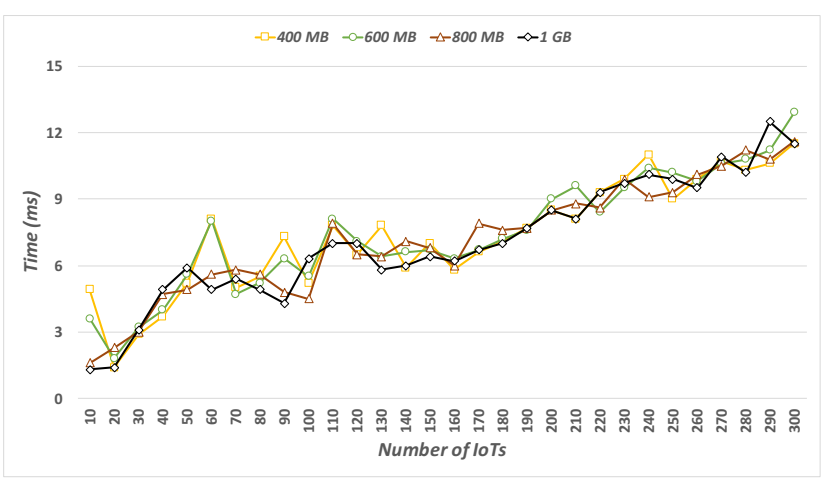

Figure 6: Comparison between number of IoT devices and execution time over different data sizes.

\subsection{Laboratory Experiments}

The next set of experiments aimed to test the performance of the proposed approach compared to real prototype data. Specifically, we consider a Human Activity Recognition (HAR) scenario, where data captured by motion sensors (e.g. accelerometer and gyroscope) within the IoT devices can be analyzed to infer user's current physical activity. In particular, personal smart devices are exploited to collect and send the sensors' raw data to the FAP for classification. In such a scenario, the IoT task submitted to the FAP is to infer the user's current physical activity via extracting feature vectors from a fixed time-window periodically, and classify the activity based on a machine learning algorithm.

The prototype of the proposed environment is depicted in Fig. 7, where a 2013 MacBook Pro Retina, a Samsung Gear S3 Frontier smartwatch, and Samsung Galaxy S7 Edge smartphone are used as FAP, IoT and hotspot, respectively. The first is characterized by a $2.4 \mathrm{GHz}$ dual-core Intel Core i5 processor, 4 GB of RAM, 128 GB of memory, and several communication capabilities, like WiFi and Bluetooth 4.0 wireless technology. The latter has an Exynos dual-core processor that works at the frequency of $1 \mathrm{GHz}, 768 \mathrm{MB}$ of RAM, Wi-Fi, and Bluetooth 4.2 communication capabilities.

The Human Activity Recognition system follows the guidelines described as in [7]. The smartwatch collects raw data with a sampling frequency of $100 \mathrm{~Hz}$ and forwards them to the FAP, over the

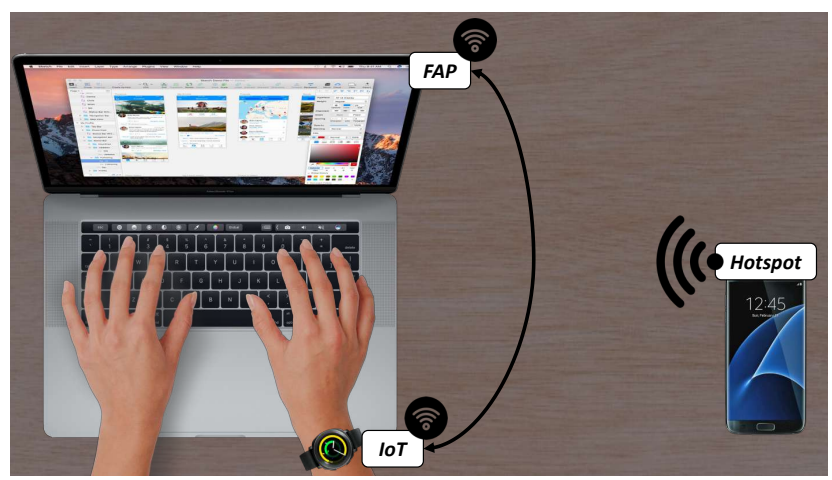

Figure 7: Test bed for the Human Activity Recognition task.

hotspot of a cellular smartphone. The FAP receives data and extract (i) max value, (ii) min value, (iii) mean, (iv) standard deviation, and (v) root mean square over the three accelerometer and gyroscope axes, within fixed-width windows of 3 seconds. Finally, the activity is classified by means of $K$-Nearest Neighbors (K-NN) algorithm and the result is sent back to the IoT device.

Fig. 8 shows that with the increase of data size total service latency increases. However, the service latency obtained in the real and numerical result is almost the same with increasing data sizes. The reason for the slight difference between real data and numerical result is due to the fact that, smart-phone and laptop used in the model are not dedicated as SAP and FAP respectively, and they were running other applications in the background at the time of data execution.

Comparison with existing work: We compared our algorithm with a state-of-art approach described in [13]. Here, authors propose a matching model based on Student Project Allocation (SPA) problem, where IoTs are considered as students who are assigned several projects under the assistance of the lecturers, i.e., service providers. The results obtained in Fig. 9 show that our algorithm reduces the average latency of the entire system by about a factor of 10 on average. This outperformance is justified by two key elements. First, in our case, the search for the best match is distributed by means that it is driven by each IoT, while in [13] it is entirely conducted by the service provider. Second, the authors propose a latency service model that tends to distribute resources equally among all IoT devices regardless of heterogeneous deadlines, limiting average service latency performance. However, sharing equal resources among different IoT's task is not a well motivating factor in the scenario where different tasks will have heterogeneous deadlines to be completed. Unlike the existing work, our model prepares the preference list of individual IoT's task and priorities the task having less required latency to be executed first. Thus, our model is more suitable for real applications where tasks will have heterogeneous deadlines to be completed.

\section{CONCLUSION}

In this paper, we address the problem of resource provisioning in the IoT environment exploiting fog computing and $5 \mathrm{G}$ cellular network 


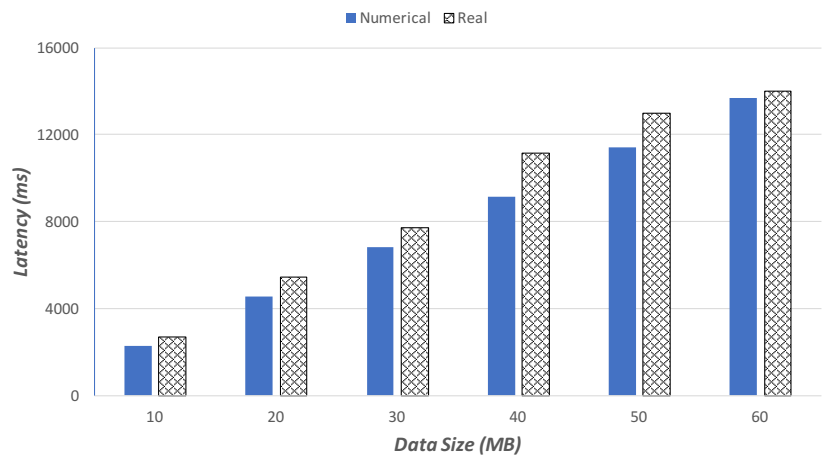

Figure 8: Comparison of service latency and data size on developed prototype and numerical result.

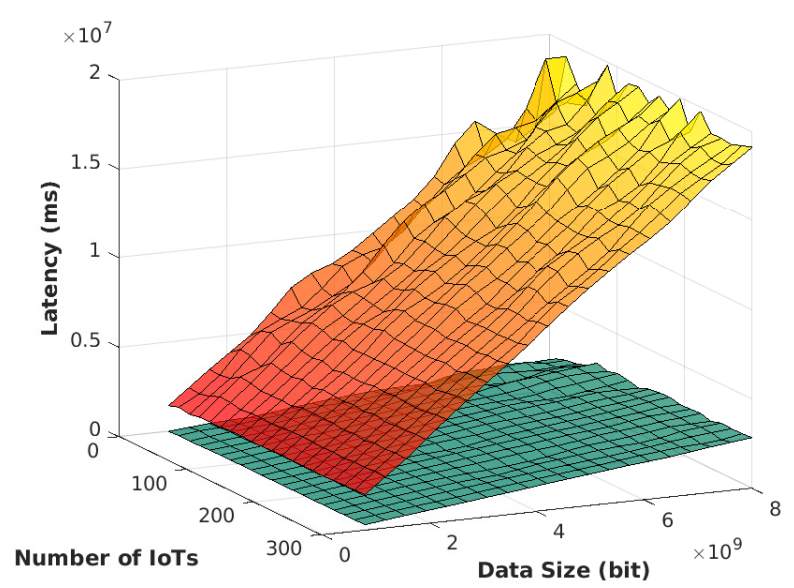

Figure 9: Comparison between proposed matching algorithm (green curve) with a state-of-art approach presented in [13] (orange curve).

model altogether. To this aim, we have formulated average service latency minimization as an optimization problem and proposed a three-sided stable matching algorithm to solve it. The extensive theoretical and experimental analysis showed the validity of the proposed scheme in terms of latency, users' satisfaction and execution time. Moreover, a comparison between our implementation and state-of-the-art techniques have been presented, to prove the effectiveness of our approach in a real scenario.

Acknowledgments: This work is partially supported by the NSF grants under award numbers CNS-1818942, CCF-1725755, CNS1545037, CNS-1545050.

\section{REFERENCES}

[1] Vangelis Angelakis et al. 2016. Allocation of heterogeneous resources of an IoT device to flexible services. IEEE Internet of Things fournal 3, 5 (2016), 691-700.

[2] Eduardo M Azevedo et al. 2016. A supply and demand framework for two-sided matching markets. Fournal of Political Economy 124, 5 (2016), 1235-1268.

[3] Flavio Bonomi et al. 2012. Fog Computing and Its Role in the Internet of Things. In Proceedings of the First Ed. of the MCC Work. on Mobile Cloud Computing (MCC '12). ACM, New York, NY, USA, 13-16. https://doi.org/10.1145/2342509.2342513

[4] Min Chen et al. 2018. Data-driven computing and caching in 5G networks: Architecture and delay analysis. IEEE Wir. Comm. 25, 1 (2018), 70-75.
[5] Francesco Chiti et al. 2018. A Matching Theory Framework for Tasks Offloading in Fog Computing for IoT Systems. IEEE Int. of Things four. 5, 6 (2018), 5089-5096.

[6] S. Choy et al. 2012. The brewing storm in cloud gaming: A measurement study on cloud to end-user latency. In 2012 11th Annual Workshop on Network and Systems Support for Games (NetGames). IEEE, Piscataway, NJ, USA, 1-6. https: //doi.org/10.1109/NetGames.2012.6404024

[7] Federico Concone et al. 2017. Smartphone Data Analysis for Human Activity Recognition. In $A I^{*} I A 2017$ Advances in Artificial Intelligence. Springer International Publishing, Cham, 58-71.

[8] Yongsheng Ding et al. 2013. An intelligent self-organization scheme for the internet of things. IEEE Computational Intelligence Magazine 8, 3 (2013), 41-53.

[9] ETSI. 2017-08. LTE; Evolved Universal Terrestrial Radio Access (E-UTRA); Physical channels and modulation (3GPP TS 36.211 version 14.3.0 Release 14).

[10] LTE ETSI. 3rd Generation Partnership Project, Sophia-Antipolis, France, 3GPP Tech. Spec. TS 36.300 ver. 10.5.0, Oct. 2011. Evolved Universal Terrestrial Radio Access (E-UTRA) and Evolved Universal Terrestrial Radio Access Network (EUTRAN); Overall Description; Stage 2.

[11] David Gale and Lloyd S Shapley. 1962. College admissions and the stability of marriage. The American Mathematical Monthly 69, 1 (1962), 9-15.

[12] Sanjeevini Devi Ganni, Ajay Pratap, and Rajiv Misra. 2017. Distributed Algorithm for Resource Allocation in Downlink Heterogeneous Small Cell Networks. In Proceedings of the 7th ACM International Workshop on Mobility, Interference, and MiddleWare Management in HetNets (MobiMWareHN'17). ACM, New York, NY, USA, Article 5, 6 pages. https://doi.org/10.1145/3083201.3083203

[13] Yunan Gu et al. 2018. Joint radio and computational resource allocation in IoT fog computing. IEEE Trans. on Veh. Techn. 67, 8 (2018), 7475-7484.

[14] Nadeem Javaid et al. 2018. Intelligence in IoT-Based 5G Networks: Opportunities and Challenges. IEEE Communications Magazine 56, 10 (2018), 94-100.

[15] Boqi Jia et al. 2018. Double-matching resource allocation strategy in fog computing networks based on cost efficiency. Journal of Communications and Networks 20, 3 (2018), 237-246.

[16] Song Li et al. 2018. Energy-efficient resource allocation for industrial cyberphysical IoT systems in 5G era. IEEE Transactions on Industrial Informatics 14, 6 (2018), 2618-2628.

[17] Adusumalli Sai Manoj et al. 2019. Patient Health Monitoring Using IoT. In Mobile Health Applications for Quality Healthcare Delivery. IGI Global, Hershey, PA, USA, $30-45$.

[18] Cheng Ng et al. 1991. Three-dimensional stable matching problems. SIAM fournal on Discrete Mathematics 4, 2 (1991), 245-252.

[19] Ajay Pratap et al. 2016. Randomized graph coloring algorithm for physical cell ID assignment in LTE-a femtocellular networks. Wireless Personal Communications 91, 3 (2016), 1213-1235.

[20] Ajay Pratap et al. 2018. Distributed Randomized $k$-Clustering Based PCID Assignment for Ultra-Dense Femtocellular Networks. IEEE Transactions on Parallel and Distributed Systems 29, 6 (2018), 1247-1260.

[21] Ajay Pratap et al. 2018. Random Graph Coloring-Based Resource Allocation for Achieving User Level Fairness in Femtocellular LTE-A Networks. Wireless Personal Communications 98, 2 (2018), 1975-1995.

[22] Ajay Pratap et al. 2018. Resource Allocation to Maximize Fairness and Minimize Interference for Maximum Spectrum Reuse in 5G Cellular Networks. In 2018 IEEE 19th International Symposium on" A World of Wireless, Mobile and Multimedia Networks"(WoWMoM). IEEE, Piscataway, NJ, USA, 1-9.

[23] Ajay Pratap et al. 2019. Maximizing Joint Data Rate and Resource Efficiency in D2D-IoT Enabled Multi-Tier Networks. In to appear in 44th IEEE LCN. IEEE, Piscataway, NJ, USA, 1-8.

[24] Ajay Pratap et al. 2019. On Maximizing Task Throughput in IoT-Enabled 5G Networks Under Latency and Bandwidth Constraints. In 2019 IEEE SMARTCOMP. IEEE, Piscataway, NJ, USA, 217-224. https://doi.org/10.1109/SMARTCOMP.2019. 00056

[25] Alvin E Roth. 2008. Deferred acceptance algorithms: History, theory, practice, and open questions. international fournal of game Theory 36, 3-4 (2008), 537-569.

[26] Surbhi Saraswa et al. 2019. Energy Efficient Data Forwarding Scheme in FogBased Ubiquitous System with Deadline Constraints. IEEE Tranactions on Network and Service Management 0, 0 (2019), 1-14.

[27] Stefania Sardellitti et al. 2015. Joint optimization of radio and computational resources for multicell mobile-edge computing. IEEE Transactions on Signal and Information Processing over Networks 1, 2 (2015), 89-103.

[28] Ashok Subramanian. 1994. A new approach to stable matching problems. SIAM 7. Comput. 23, 4 (1994), 671-700.

[29] Huaqing Zhang et al. 2017. Computing resource allocation in three-tier IoT fog networks: A joint optimization approach combining Stackelberg game and matching. IEEE Internet of Things fournal 4, 5 (2017), 1204-1215.

[30] W. Zirwas, R. SivaSiva Ganesan, and B. Panzner. 2018. Sub Tiling - a flexible CSI Reference Signal Concept for 5G New Radio Systems. In WSA 2018; 22nd International ITG Workshop on Smart Antennas. IEEE, Piscataway, NJ, USA, 1-5. 